Aus der Abteilung für Ohren-, Nasen- und Halsleiden des Städtischen Luisenhospitals in Dortmund. (Dirigierender Arzt: Oberarzt Dr. Hansberg.)

\section{Vollständige sensorische Aphasie bei Läsion der rechten ersten Schläfenwindung.}

Von Dr. Wilhelm Meyer, früher Assistent der Abteilung, jetzt Spezialarzt für Ohrenleiden in Kassel.

Völlige sensorische Aphasie infolge Läsion des rech te n Schläfenlappens bei einem Rechtshänder ist ein so seltener, ungewöhnlicher, bei diagnostisch-therapeutischen Ueberlegungen unter Umständen irreführender und für den Kranken verhängnisvoller Befund, daß jeder diesbezügliche Fall mir der Veröffentlichung wert erscheint.

Krankengeschichte: Ein 26jähriger Arbeiter kommt am 20. September 1906 allein ins Luisenhospital in Dortmund, ist aber nicht imstande, sich zu verständigen. Er kann nur einige unverständliche Laute sprechen, versteht keine Fragen, befolgt keine Aufforderung, gibt nur durch Zeichen zu verstehen, daß ihn die rechte Kopfseite schmerze. Aufnahme auf die Chirurgische Abteilung; äußerer Befund negativ außer einer Temperatur von $38,5^{\circ}$. Nach 2 Tagen übelriechender Ausfluß aus dem rechten Ohr, weswegen Jodoform in den Gehörgang geblasen wird. Darnach ausgedehntes Ekzem der Ohrmuschel und ihrer Umgebung mit völliger Verschwellung des Gehörganges, weshalb Patient der Ohrenabteilung überwiesen wird, nachdem der zugezogene Oberarzt der Inneren Abteilung (Dr. Vollhard) die Meinung geäußert hatte, es könne sich vielleicht um einen AbszeB im rechten Schläfenlappen handeln.

Befund am 22. September 1906: Patient kann ohne Unterstiutzung gehen, den Kopf frei nach allen Richtungen bewegen und sich bücken, anscheinend ohne Schmerzen. Am Kopfe nirgends Spuren einer Verletzung nachweisbar. Innere Organe gesund; Augenhintergrund normal. Kopfnerven frei bis auf eine leichte rechtseitige Facialisparese, doch ist eine Beteiligung der Stirnäste nicht sicher festzustellen. Akutes Ekzem der Ohrmuschel mit starker ödematöser Schwellung vor und hinter derselben; völlige Verschwellung des Gehörganges, übelriechende Sekretion aus demselben. Durch Katheterismus wird Perforation des rechten Trommelfells festgestellt; linkes Ohr gesund; Temperatur 38,0, Puls 80. Patient versteht kein Wort, befolgt keine Aufforderung, z. B. die Zunge herauszustrecken, die Stirn kraus zu machen, selbst wenn es ihm vorgemacht wird. Er liest nur kurze Worte, z. B. „in“, „zwar", von zusammengesetzten nur die Anfangsbuchstaben. "Den Namen von ihm vorgehaltenen Gegenständen wie Schlüssel, Messer, Bleifeder, kann er nicht angeben, kennt ihre Bedeutung nicht, murmelt nur undeutliche Worte. Von ihm vorgeschriebenen Worten schreibt er nur die Anfangsbuchstaben nach. Beim Schreiben, Ankleiden, Essen bedient er sich der rechten Hand.

Vom 23.-27. September keine Aenderung im Befunde; die Temperatur bewegte sich ständig um 38,0.

Die Diagnose schwankte zwischen HirnabszeB und Bluterguß, doch wird letzterer mit größerer Wahrscheinlichkeit angenommen, wobei es zweifelhaft bleibt, ob der Herd rechts oder links sitzt.

Am 27. September Operation: Eröffnung des rechten Warzenfortsatzes; die Zellen sind blutig gefärbt, in der Tiefe eine Fissur des Schläfenbeins, welche in einer Länge von etwa $5 \mathrm{~cm}$ von hinten oben nach vorn unten bis in die mit Eiter gefüllte Pauke geht; im hinteren unteren Quadranten des Trommelfells ein etwa $4 \mathrm{~mm}$ langer Riß; in der Gegend der ersten Schläfenwindung ein etwa $4 \mathrm{~cm}$ langes und breites Hämatom der Dura; die Dura selbst stellenweise blutig imbibiert und zerrissen. Dura wird bis ins Gesunde freigelegt.

Einige Tage nach der Operation Fieberlosigkeit; die Aphasie war nach 14 Tagen, die Facialisparese nach 4 Wochen geschwunden. Am 15. März 1907 wurde Patient mit vernarbtem Trommelfell und gutem Gehör als völlig geheilt entlassen. Die Anamnese wird jetzt vom Patienten folgendermaßen angegeben: Am 17. September 1906 in angetrunkenem Zustande Sturz von der Treppe; hiernach bemerkte er Brausen im Kopf, ging die Treppe wieder hinauf, legte sich zu Bett, schlief, bemerkte am anderen Morgen beim Erwachen, daß Blut aus dem rechten Ohre geflossen war, daß er nicht gut sprechen konnte. In der nächsten Nacht kein Schlaf wegen Ohren sausen und Kopfschmerzen rechterseits. Am nächsten Tage ging Patient zu seiner Krankenkasse, konnte aber nicht die Stelle des Werkes (5000 Arbeiter) bezeichnen, wo er arbeitete, fand die Worte dafür nicht, obwohl er wußte, wo die Stelle war. Er versuchte durch Zeichen, an der Kasse einen Aufnahmeschein für das Krankenhaus zu bekommen; da die Beamten ihn nicht verstanden, kam er ohne Papiere allein ins Krankenhaus.
Nach dem Operationsbefunde lag also - neben der Ruptur des rechten Trommelfells und der sekundären rechtseitigen Mittelohreiterung - eine Fraktur der rechten Felsenbeinpyramide mit einem auf die erste Schläfenwindung des Gehirns drückenden Hämatom der Dura vor.

War dieser Befund schon vor der Operation mit Sicherheit zu diagnostizieren? Nein. Aeußere Zeichen einer Kopfverletzung konnten auf der Ohrenstation nicht festgestellt werden; weder auf Fragen nach einer vorausgegangenen Verletzung noch nach dem Alter der Mittelohreiterung konnte der aphatische Patient Auskunft geben.

Eine Oeffnung im rechten Trommelfell konnte zwar mittels des Katheters hergestellt werden; zweifelhaft blieb aber infolge der völligen Verschwellung des Gehörgangs, ob es sich um eine traumatische Ruptur oder um eine frische oder alte Ohreiterung handelte.

Aus demselben Grunde blieb es auch ungewiß, ob die Facialisparese etwa durch einen eitrigen Prozeß im rechten Mittelohr bedingt war. $\mathrm{Ob}$ sie eine peripherische oder zentrale war, mußte bei der Undeutlichkeit des Befallenseins der Stirnäste unentschieden bleiben; eine eingehende Prüfung scheiterte an der schweren Fixierbarkeit des Kranken. Auch eine nachweisbare leichte Beteiligung der Stirnäste konnte auf eine linkseitige zentrale Affektion bezogen werden, wie es - wenn auch selten - bei Kopfverletzungen beobachtet ist.

Es war also mindestens zweifelhaft, ob die Facialisparese durch eine peripherische rechtseitige oder linkseitige zentrale Affektion bedingt war. Auf letztere mußte nun das Symptom der sensorischen Aphasie hinweisen, welches ja als charakteristisch und pathognomonisch für eine Läsion des linken Schläfenlappens gilt und be Blutungen, Abszessen oder Tumoren an dieser Stelle häufig beschrieben ist.

Die ständige niedrige Temperatur sprach nicht gegen einen Abszeß, wohl aber der Umstand, daß völlige Aphasie dabei sehr selten ist, daß Patient während der siebentägigen Beobachtung keinen schwerkranken Eindruck machte, wie man bei einem Hirnabszeß zu sehen gewohnt ist. Auch fehlte für einen linkseitigen Abszeß der Ausgangspunkt, indem Spuren von einer Schädelverletzung, Knochenaffektion, einer frischen oder alten Ohreiterung linkerseits nicht vorhanden waren.

Einen metastatischen, von der rechtseitigen Ohreiterung ausgegangenen AbszeB linkerseits anzunehmen, erschien uns recht gezwungen.

Nahm man eine rechtseitige intrakranielle Komplikation der Ohreiterung an, erschien wohl die Facialisparese, nicht aber die Aphasie hiermit vereinbar, da ja Patient $R$ echtshänder war und nach der üblichen Lehre nur bei Linkshändern das Sprachzentrum im rechten Schläfenlappen liegen sollte.

Am wahrscheinlichsten erschien uns immer noch ein Hämatom der Dura infolge einer Verletzung, wenn auch sein Sitz zweifelhaft blieb.

Bei den schweren Symptomen mußte etwas geschehen; ein Hirnabszeß mußte entleert, ein rechtseitiges Hämatom mußte freigelegt und entfernt werden, drohte doch die Gefahr der Vereiterung desselben mit anschließender Meningitis von der eiternden Paukenhöhle her.

Eine beiden Erwägungen Rechnung tragende Trepanation ent schlossen wir uns rechtseitig vorzunehmen, da der Kranke ja seine rechte Kopfseite als schmerzhaft angedeutet hatte, rechterseits auch eine Ohreiterung und Facialisparese vorhanden war, trotzdem uns das Hauptsymptom der Aphasie bei dem Rechtshänder eigentlich zu einer Trepanatiolı der linken Schädelhöhle hätte veranlassen müssen; hat doch ein Operateur in demselben Jahre - wie wir nachträglich aus der Literatur ersahen - bei einem rechtseitigen otitischen Schläfenlappenabszeß eines Rechtshänders im Vertrauen auf die hier auch vorhandene sensorische Aphasie links trepaniert und erst bei der Sektion den Eiterherd im rechten Schläfenlappen gefunden.

Glücklicherweise trafen wir mit der rechtseitigen Trepanation das erwartete Hämatom der Dura.

Können wir nun aus dem Erfolge der Operation, der Wiederkehr der Sprache nach 14 Tagen schließen, daß das Hämatom au dem rechten Schläfenlappen die sensorische Aphasie verursucht hat, obwohl es sich hier nicht um einen Linkshänder handelte?

Ich glaube diese Frage mit Wahrscheinlichkeit bejahen zu können. Der Einwand, daß Patient vielleicht doch Linkshänder war, erscheint mir nicht zulässig, da wir selbst häufig beobachtet haben wie er sich bei den Verrichtungen des täglichen Lebens in üblicher Weise der rechten Hand bediente, da er selbst nach seiner Genesung auf unsere Fragen stets angab, nie „links" gewesen zu sein.

Auch ist im linken Schläfenlappen sicher kein Abszeß oder Tumor vorhanden gewesen, wie die andauernde Gesundheit des Mannes in der Folgezeit bewiesen hat. Eine Möglichkeit zwar, daß 
bei dem Sturz von der Treppe auch im linken Schläfenlappen Blutergüsse erfolgt seien, welche spontan resorbiert sind, ist ja nicht auszuschließen. Eine derartige Möglichkeit könnte übrigens nur eine Sektion und die mikroskopische Untersuchung der betreffenden Hirnpartie ausschließen.

Wenn schließlich auch Herz und Blinddarm einmal ausnahmsweise in der der üblichen entgegengesetzten Körperhälfte bei sonst normalen Leuten liegen können, warum soll dann nicht auch einmal das Sprachzentrum bei einem Rechtshänder eine Ausnahme von der Regel machen können? Von dieser Ausnahme fand ich bei einer nachträglichen Durchsicht der mir zugănglichen Literatur vier Fälle beschrieben - ob noch mehrere veröffentlicht sind, muß ich dahingestellt sein lassen - von Joffroy (1), Forselles (2), Heine (3), Wittmaack (4), auf welche ich den Leser des näheren verweisen muß.

Sämtliche Kranke waren zweifellos Rechtshänder. Bei dem Kranken von Joffroy handelte es sich um eine apoplektische Blutung, bei den übrigen um otitische Abszesse; bei allen Kranken fanden sich die Herde im rechten Schläfenlappen, bei allen fand sich das Symptom der senorischen Aphasie. In den zur Sektion gelangten Fällen von J offroy und Wittmaack wurde im linken Schläfenlappen nichts gefunden, was die sensorische Aphasie hätte erklären können.

Es dürfte aus diesen Fällen wie aus dem unsrigen hervorgelıen, daß 3 auch bei notorischen Rechtshändern ausnahmsweise das Sprachzentrum im rechten statt im linken Schläfenlappen liegen kann, daß ferner die sensorische Aphasie auch bei Rechtshändern nicht immer, wie es gleichsam als Dogma noch in den neuesten und gangbarsten Lehrbüchern zu lesen steht, ein sicheres, pathognomonisches Symptom für eine Affektion im linken Schläfenlappen sein muß.

Die Erinnerung an die erwähnten Ausnahmefälle dürfte geeignet sein, Chirurgen und Ohrenärzte vor diagnostischen Irrtümern und Eingriffen an falscher Stelle zu bewahren, ihnen den EntschluB zu erleichtern, bei ähnlichen Gelegenheiten die rechte Schädelhöhle zu erőffnen, wenn sich außer der Aphasie bei einem Rechtshänder Symptome vorfinden, welche auf eine Affektion im linken Schläfenlappen ungezwungen nicht bezogen werden können.

Nebenbei erinnert unser Fall an die wichtige, oft vernachläßigte Mahnung, bei unklaren Schädelaffektionen sofort die Ohren zu untersuchen. Uns wäre die Schwierigkeit in der Deutung der vorliegenden Affektion erheblich erleichtert worden, wenn gleich bei der Aufnahme des Kranken ins Hospital durch den Ohrenspiegel die frische Ruptur des Trommelfells - vor dem Eintritt der sekundären Mittelohreiterung - festgestellt wäre, wodurch wenigstens die Aetiologie von vornherein geklärt gewesen wäre.

Li teratur: 1. Ref. Neurologisches Zentralblatt 1903. - 2. Ref. Archiv für Ohrenheilkunde, Band 68. - 3. Verhandlungen der Deutschen otologischen Gesellschaft 1903 - 4. Archiv für Ohrenheilkunde, Band 73. 